\title{
Hydrodynamic Instabilities in the Models of the Formation of Young Stellar Objects
}

\author{
G.Yu. Kotova1 ${ }^{1}$, K.V. Krasnobaev ${ }^{1,2}$ \\ ${ }^{1}$ Faculty of Mechanics and Mathematics, Lomonosov Moscow State University, \\ Moscow, 119991, Russia \\ ${ }^{2}$ Space Research Institute of the Russian Academy of Sciences (IKI), \\ Moscow 117997, Russia \\ kvk-kras@list.ru,gviana.k@gmail.com
}

\begin{abstract}
The features of unstable motions in the regions of active star formation are considered. The purpose of the research is to determine the magnitude and scale of inhomogeneities for which the Jeans gravitational compression criterion is satisfied. Mathematical models are used that take into account nonequilibrium radiation processes and self-gravity. The results of calculations of the cumulation of mass during the propagation of the ionization-shock front in the cloud are presented; a qualitative agreement is established between the motion structure and the one obtained on the basis of the thin layer model Cherny. In the case of Richtmyer-Meshkov instability it was found that density perturbations increase significantly due to radiation cooling. Taking into account the gravitational interaction between particles of the accelerating moving gas layer, a criterion is formulated for the dominant influence of self-gravity on the shape of inhomogeneities compared with the Rayleigh-Taylor instability. Morphologically, heterogeneities of various origins differ little and are observed mainly on the inner side of the ionization-shock front, facing the radiation source. The authors of this work for the first time found that gas-dynamic phenomena at the outer boundary of a layer compressed by a shock wave can reflect the presence of condensations that existed in the interstellar medium before the passage of the ionizationshock front. Computer modeling has shown that when a condensation (cloud, clot) penetrates into an accelerating moving layer, the formation of a cumulative jet occurs. The effect of acceleration, gas densities in the layer and in the cloud, and the shape of the cloud on the manifestation of the cumulative effect is investigated.
\end{abstract}

Keywords: hydrodynamics - HII regions - instabilities 


\title{
Гидродинамические неустойчивости в моделях образования молодых звездных объектов
}

\author{
Г.Ю. Котова ${ }^{1}$, К.В. Краснобаев ${ }^{1,2}$ \\ ${ }^{1}$ Механико-математический факультет МГУ имени М.В. Ломоносова, \\ Россия, Москва, 119991, Ленинские горы ГСП-1 \\ ${ }^{2}$ Институт космических исследований РАН, \\ Россия, Москва, 117997, Профсоюзная 84/32 \\ kvk-kras@list.ru,gviana.k@gmail.com
}

\begin{abstract}
Аннотация
Рассматриваются особенности неустойчивых движений в областях активного звездообразования. Цель исследований заключается в определении величины и масштабов неоднородностей, для которых выполняется критерий гравитационного сжатия Джинса. Используются математические модели, учитывающие неравновесные радиационные процессы и самогравитацию. Представлены результаты расчетов кумуляции массы при распространении ионизационно-ударного фронта в облаке, установлено качественное согласие структуры движения с получаемой на основе модели тонкого слоя Г.Г. Черного. В случае неустойчивости Рихтмайера-Мешкова найдено, что возмущения плотности существенно увеличиваются из-за радиационного охлаждения. С учетом гравитационного взаимодействия между частицами ускоренно движущегося газового слоя сформулирован критерий доминирующего влияния самогравитации на форму неоднородностей по сравнению с неустойчивостью Рэлея - Тейлора. Морфологически неоднородности различного происхождения отличаются мало и наблюдаются преимущественно на внутренней - обращенной к источнику излучения - стороне ионизационно-ударного фронта. Авторами настоящей работы впервые установлено, что газодинамические явления на внешней границе сжатого ударной волной слоя могут отражать присутствие уплотнений, которые существовали в межзвездной среде до прохождения ионизационно-ударного фронта. Компьютерное моделирование показало, что, когда уплотнение (облако, сгусток) проникает в ускоренно движущийся слой, происходит формирование кумулятивной струи. Исследовано влияние величины ускорения, плотностей газа в слое и в облаке, формы облака на проявление кумулятивного эффекта.
\end{abstract}

Ключевые слова: гидродинамика - области НІІ - неустойчивости

\section{1. Введение}

В настоящее время газодинамические модели широко применяются для решения проблемы звездообразования, которая является одной из важнейших в астрофизике. Так, хорошо известна триггерная модель образования звезд [1]. Согласно модели, молодые звезды с массами $\sim 10 M_{\odot}$ и с температурами $\sim(3 \div 4) \times 10^{4} \mathrm{~K}$ нагревают и ионизуют окружающую их межзвездную среду. Образуются области практически полностью ионизованного водорода области HII. При расширении нагретого газа на периферии области НII возникает плотная оболочка, ограниченная ударной волной и фронтом ионизации - ионизационно-ударный или I-S фронт. По мере распространения ионизационно-ударного фронта масса оболочки увеличивается и создаются условия для развития неустойчивости Джинса и появления нового поколения звезд. 
Модель подтверждается исследованиями с помощью космических аппаратов «Spitzer», «Herschel», «Planck». Выявлено существование сотен расширяющихся областей HII, окруженных молодыми звездными объектами [2-5]. Эти объекты часто располагаются в уплотнениях, процессы возникновения и эволюции которых определяют темп звездообразования. Происхождение неоднородностей объясняется развитием гравитационной неустойчивости или неустойчивости ионизационно-ударного фронта, а также проникновением в область НІІ хаотически распределенных возмущений из межзвездной среды. Трудность идентифицировать тот или иной сценарий заключается в том, что образуются структуры, морфологически мало отличающиеся между собой [6-8]. Кроме того, наблюдаемые «узелки», «пальцы», «столбики», «глобулы» располагаются преимущественно в окрестности внутренней - обращенной к центральной звезде - границе плотного слоя нейтрального газа, возникающего вследствие расширения области НІІ. В то же время взаимодействие уплотнений с внешней границей слоя исследовано значительно меньше. Поэтому в настоящей работе рассматривается одно из проявлений такого взаимодействия, которое заключается в формировании кумулятивных струй при проникновении уплотнения (облака, сгустка) в ускоренно движущийся слой.

План работы следующий. В разделе 2 излагается общий подход к моделированию образования плотных слоев и оболочек, дается математическая формулировка задачи о расширении области НII. Раздел 3 содержит примеры расчета эволюции возмущений в излучающей оболочке при развитии гравитационной неустойчивости, неустойчивостей I-S фронта и Рихтмайера-Мешкова. Моделированию проникновения облака в ускоренно движущийся слой посвящен раздел 4. Обсуждение результатов проводится в разделе 5.

\section{2. Модели расширения области Н ІІ. Образование плотных слоев и оболочек}

Классической теории расширения областей НІІ посвящен целый ряд обзоров и монографий (например, [9-11]). Основными физическими процессами, определяющими тепловое и ионизационное состояние, являются: нагрев и ионизация газа ультрафиолетовым излучением центральной звезды (или группы звезд) и потери энергии в результате фоторекомбинаций и возбуждения с последующим высвечиванием уровней наиболее обильных ионов тяжелых элементов (азота, кислорода, углерода и др.). Замкнутая система уравнений, описывающих движение трехкомпонентной плазмы в области НII, имеет вид

$$
\begin{gathered}
\frac{\mathrm{d} \rho}{\mathrm{d} t}+\rho \operatorname{div}(\mathbf{V})=0, \quad \rho \frac{\mathrm{d} \mathbf{V}}{\mathrm{d} t}+\operatorname{grad} p=0, \\
\frac{\mathrm{d} E}{\mathrm{~d} t}+\frac{p}{\rho} \operatorname{div}(\mathbf{V})=\frac{\Gamma-\Lambda}{\rho}, \quad \frac{\mathrm{d} s}{\mathrm{~d} t}=(1-s) \int_{v_{H}}^{\infty} \int_{4 \pi} \sigma_{H v} \frac{I_{v}}{h v} \mathrm{~d} v \mathrm{~d} \omega-\frac{s^{2} \rho}{m_{H}} \alpha_{H}(T), \\
\frac{\mathrm{d} I_{v}}{\mathrm{~d} l}=\alpha_{v} I_{v}+\varepsilon_{v}, \quad \mathrm{E}=\frac{1}{\gamma-1} \frac{p}{\rho}, \quad \Gamma=\int_{v_{H}}^{\infty} \int_{4 \pi} \alpha_{v} I_{v} \mathrm{~d} v \mathrm{~d} \omega, \quad \Lambda=4 \pi \int_{0}^{\infty} \varepsilon_{v} \mathrm{~d} v,
\end{gathered}
$$

где искомыми функциями пространственных координат и времени $t$ являются плотность $\rho$; скорость $\mathbf{V}$, давление $p$; степень ионизации $s=n_{H+} /\left(n_{H}+n_{H+}\right)$; интенсивность излучения $I_{v}\left(v-\right.$ частота квантов); $v_{H}$ - частота ионизации водорода; $\omega$ - телесный угол; $\alpha_{v}$ и $\varepsilon_{v}-$ соответственно коэффициенты поглощения и излучения; $l$ - расстояние вдоль траектории фотона; $\sigma_{H v}$ - сечение фотоионизации; $h$ - постоянная Планка; $\alpha_{H}(T)$ - коэффициент фоторекомбинации; $\alpha_{v}=\sigma_{H v} n_{H}$. Величины Г и $\Lambda$-энергия, соответственно приобретаемая и теряемая единичным объемом газа в единицу времени. В (1) предполагается, что плазма ква- 
зинейтральна (т.е. $n_{H+}=n_{e}$, где $n_{H}, n_{H+}, n_{e}$ - соответственно концентрации нейтрального водорода, протонов и электронов); показатель адиабаты $\gamma=5 / 3$, и вкладом малых примесей тяжелых элементов в выражениях для $\rho$ и $p$ можно пренебречь, так что $\rho=m_{H}\left(n_{H+}+n_{H}\right)$, $p=(1+s) \rho k T / m_{H} \quad\left(m_{H}\right.$ - масса атома водорода; $T$ - температура, $k$ - постоянная Больцмана). Обычно в расчетах в начальный момент времени задается пространственное распределение искомых функций, а граница расчетной области выбирается на достаточно большом расстоянии от звезды, где параметры среды не возмущены.

Система уравнений (1) весьма сложна и часто для описания динамики областей HII используются приближенные модели: для «медленных» движений - приближение изотермичности, для «быстрых» - адиабатичности, для движения слоя или оболочки - приближение тонкого слоя $([10,12,13])$. В зависимости от конкретных особенностей рассматриваемого движения в разделах 3 и 4 используются как расчеты на основе полной системы уравнений, так и в рамках приближенных моделей.

Отметим также, что плотные слои могут возникать и в центральных частях областей HII, если звезда обладает звездным ветром. В этом случае при торможении звездного ветра образуются две ударные волны и контактный разрыв между ними, разделяющий вещество звездного ветра и плазму области НII.

Обратимся теперь к особенностям проявления типичных для расширения областей НІІ неустойчивостей - неустойчивости ионизационно-ударного фронта, неустойчивости Рихтмайера-Мешкова и гравитационной неустойчивости.

\section{3. Проявления неустойчивостей: неустойчивости I-S фронта и Рихтмайера-Мешкова, гравитационная неустойчивость}

Условия, в которых формируются области НII, очень разнообразны. Отличаются как характеристики звезды (ее температура, поток ионизующей радиации, спектральный состав излучения), так и параметры окружающей звезду среды (плотность и температура частиц, их пространственное распределение). Поэтому ограничимся некоторыми типичными постановками задач и получаемыми при их решении результатами. Основное внимание уделяется морфологическим особенностям неустойчивых возмущений и увеличению плотности в них.

Рассмотрим сначала качественную картину формирования ускоренно движущейся нейтральной оболочки в случае, когда сферически симметричный I-S фронт распространяется в облаке, граница которого является контактным разрывом $[14,15]$. Ударная волна, опережающая фронт ионизации, достигнув границы облака, взаимодействует с контактным разрывом. В результате возникают три поверхности разрыва - фронт ионизации, контактный разрыв и распространяющаяся перед ним преломленная ударная волна. Соответственно ускоренная оболочка располагается между ударной волной и контактным разрывом.

Исследование развития возмущений в оболочке проводилось в рамках полной системы уравнений (1).

Обозначим через $t_{\text {exit }}$ момент времени, отвечающий выходу ударной волны на границу облака. Координаты внутренней и внешней границ оболочки полагаем соответственно равными $r_{\text {int }}$ и $r_{\text {exit }}$. При $t=t_{\text {exit }}$ зададим начальные возмущения в виде

$$
\rho=\rho\left(r, t_{\text {exit }}\right), E=E\left(r, t_{\text {exit }}\right), s=s\left(r, t_{\text {exit }}\right),
$$

при $r<r_{i n t}, r>r_{\text {exit }}: v_{r}=v_{r}\left(r, t_{\text {exit }}\right), v_{\theta}=0$,

$$
\text { при } r_{\text {int }}<r<r_{\text {exit }}: v_{r}=v_{r}\left(r, t_{\text {exit }}\right)-0.1 \cos \left(\frac{2 \pi \theta}{\lambda}\right), v_{\theta}=-0.1 \sin \left(\frac{2 \pi \theta}{\lambda}\right) \text {, }
$$


где $r, \theta$ - сферические координаты; $v_{r}$ и $v_{\theta}$ - компоненты вектора скорости; $\lambda$ - постоянная, характеризующая масштаб возмущения; $r_{i n t}$ - координата I-фронта, условно определяемая при значении степени ионизации порядка $0.1 ; r_{\text {exit }}$ - радиус границы облака.

Отметим, что аналогичные начальные условия отвечают нарастающим возмущениям в классической теории неустойчивости Рэлея-Тейлора. Если толщина оболочки мала по сравнению с масштабом возмущения, то образуются конденсации вытянутой в радиальном направлении формы [16]. Но учет фотоионизации, нагрева высокоэнергичными квантами и высвечивания существенно усложняет структуру неоднородностей. Кроме того, отток массы от нейтральной оболочки вызывает дополнительное ускорение, обусловленное «реактивным» эффектом уносимого вещества.

Обратимся к результатам расчетов конкретного движения. В соответствии с наблюдениями примем, что область НІІ формируется в первоначально неподвижном облаке с концентрацией частиц $\rho_{0} / m_{H}=1 \mathrm{~cm}^{-3}$. Облако окружено средой с $\rho_{1} / m_{H}=0.1 \mathrm{~cm}^{-3}$ и имеет температуру $T_{1}=1.67 \times 10^{3} \mathrm{~K}$. Температура звезды $T_{*}=3.09 \times 10^{4} \mathrm{~K}$, a ее радиус $r_{*}=7.6 R_{\odot} . \mathrm{B}$ качестве характерного размера примем величину $r_{s t}$, близкую к радиусу облака $r_{c 0}$. Введем нормированные величины

$$
\bar{\rho}=\frac{\rho}{\rho_{0}}, \overline{\mathbf{V}}=\frac{\mathbf{V}}{a_{T}}, \bar{T}=\frac{T}{T_{E}}, \bar{r}=\frac{r}{r_{s t}}, \bar{t}=\frac{t a_{T}}{r_{s t}}, a_{T}^{2}=\frac{2 k T_{E}}{m_{H}}, T_{E}=10^{4} \mathrm{~K}
$$

Эволюции возмущений концентрации нейтральной оболочки представлена на рис. 1 для $r_{c 0}=1.2 r_{s t}$ (используются безразмерные переменные).

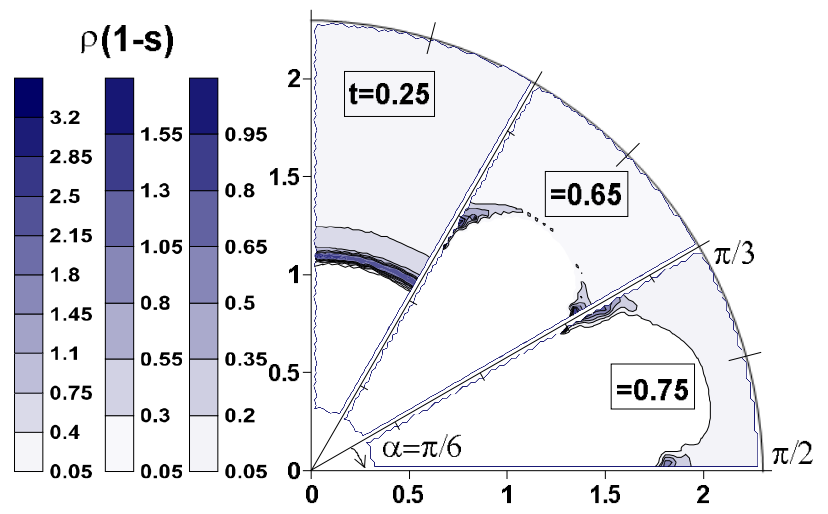

Рис.1. Изменение плотности нейтрального газа со временем в координатах $(r, \theta)$

В области $\theta \approx \pi / 3$ формируется уплотнение нейтрального газа в виде тонкого заостренного по направлению к источнику излучения стрежня, расширяющегося на противоположном конце. К моменту времени $t=0.75$ происходит разрыв оболочки - ее вещество становится полностью ионизованным и горячий газ фонтанирует в разреженную среду. Таким образом, морфология неоднородностей существенно отличается от характерной для адиабатических движений.

Радиационные процессы также являются важными, когда исследуется движение плотных слоев, порождаемых в областях НІІ торможением звездного ветра. В результате торможения образуются две ударные волны и располагающийся между ними контактный разрыв [17]. Нестационарность истечения звездных ветров может сопровождаться возникновением вторичных ударных волн. Тогда при взаимодействии этих волн с контактной поверхностью следует ожидать возбуждения неустойчивости Рихтмайера-Мешкова. В целях моделирования роста двумерных возмущений численно интегрировалась система (1). Принималось во внимание, что плазма полностью ионизованная и ее оптическая толщина мала. В таком случае правая часть уравнения притока тепла упрощается и принимает вид $-\left(\rho / m_{H}^{2}\right) \Lambda^{*}(T)$, где 
$\Lambda^{*}$ определяется, в частности, потерями энергии на высвечивание в линиях примесных элементов в высоких стадиях ионизации [17].

Пусть индексы «1» и «2» отвечают параметрам плазмы соответственно за фронтами ударных волн в звездном ветре и в области Н II. Так как температура в области «1» значительно превышает температуру в области «2», то под воздействием вторичных ударных волн контактный разрыв может быть неустойчивым. Действительно, расчеты показали, что малое гармоническое возмущение формы поверхности контактного разрыва нарастает со временем.

Введем декартовы координаты $(x, y)$ с уравнением невозмущенной контактной поверхности $x_{D}(y, 0)=0$. После столкновения приходящей из области «1» ударной волны с контактным разрывом в среде с большей плотностью будет распространяться со скоростью $u_{s}$ преломленная ударная волна. Определим далее характерное время высвечивания $t_{c}$

$$
t_{c}=\frac{p_{20}}{(\gamma-1)\left(\rho_{20} / m_{H}\right)^{2} \Lambda^{*}\left(T_{20}\right)},
$$

где $p_{20}, \rho_{20}, T_{20}$ - давление, плотность и температура в невозмущенной прохождением ударной волны области «2». Соответственно определяется и характерный пространственный масштаб движения $l_{c}=u_{s} t_{c}$.

Для оценки параметров в областях «1» и «2» воспользуемся данными из работы [17]. Полагаем

$$
n_{10}=\frac{\rho_{10}}{m_{H}}=0.0735 \mathrm{~cm}^{-3}, \quad T_{10}=2.16 \times 10^{7} \mathrm{~K} \quad \text { и } \quad n_{20}=\frac{\rho_{20}}{m_{H}}=2.57 \mathrm{~cm}^{-3}, \quad T_{20}=6.14 \times 10^{5} \mathrm{~K},
$$

и скорость падающей из области «1» ударной волны $V$ равной $V=1.15 \times 10^{8} \mathrm{~cm} \cdot \mathrm{c}^{-1}$.

Введем теперь в начальный момент времени возмущения вида

$$
x_{D}(y, 0)=A_{0} \cos \left(\frac{2 \pi y}{l}\right), A_{0}=0.4 l_{c}, l=4 l_{c}
$$

О влиянии высвечивания на структуру возмущений свидетельствует рис. 2 , на котором для моментов времени $t=3 t_{c}-(\mathrm{a}, \sigma)$ и $t=5 t_{c}-($ в, г) показаны изохоры поля течения с учетом высвечивания - (а, в) и без него - $(б, \Gamma)$. На рис. 2 значения плотности и координат $x, y$ выражены соответственно в единицах $\rho_{10}$ и $l_{c}$. Для сравнения с одномерным вариантом слева от графиков приведены также изохоры в случае $x_{D}(y, t)=x_{D}(t), x_{D}(0)=0$.
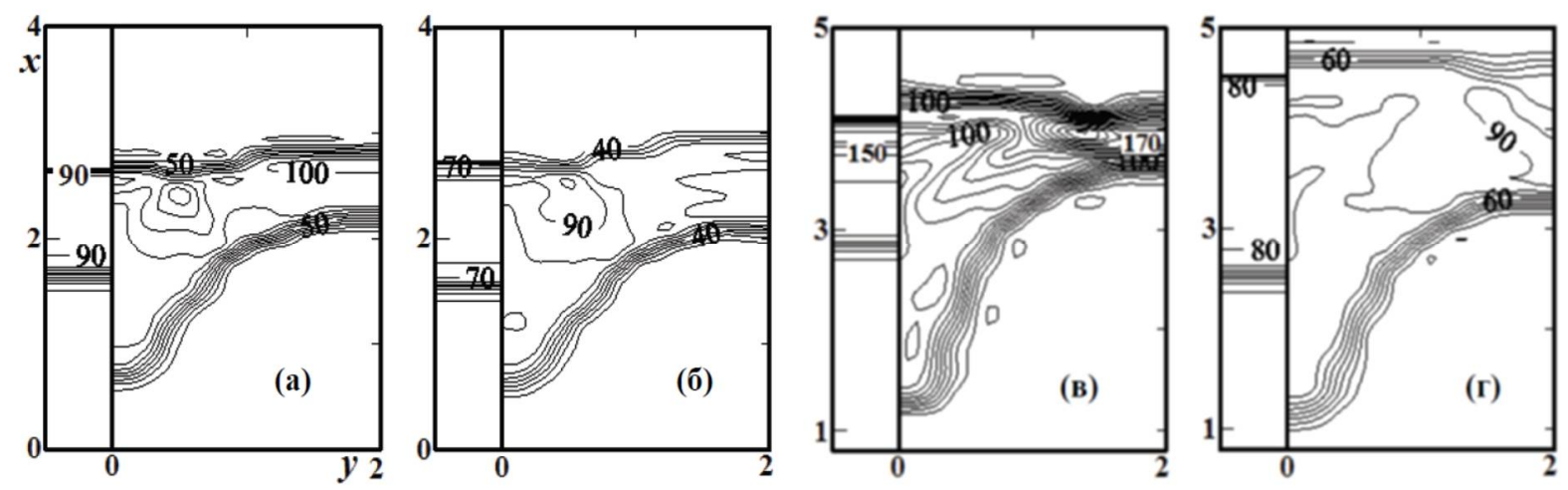

Рис. 2. Изохоры для деформированной контактной поверхности

Видно, что высвечивание мало влияет на скорость роста возмущений, в то время как плотность газа за преломленной ударной волной сильно увеличивается. 
Если неоднородности плотности, образовавшиеся в результате неустойчивостей ионизационно-ударного слоя или Рихтмайера-Мешкова, таковы, что для них выполняется критерий Джинса, то моделирование их дальнейшей эволюции требует учета самогравитации. Для этого систему (1) следует дополнить уравнением для нахождения гравитационного потенциала. Данное обстоятельство значительно усложняет моделирование неустойчивых движений и в настоящее время учет самогравитации является предметом многочисленных исследований. Применительно к условиям в оболочках на периферии областей Н II в целях упрощения расчетов воспользуемся простейшей моделью идеального совершенного газа и рассмотрим развитие возмущений в плоском слое ускоренно движущегося плоского слоя газа [18-20].

Плоские двумерные движения описываются уравнениями

$$
\frac{\partial \rho}{\partial t}+\operatorname{div}(\rho \mathbf{V})=0, \quad \rho \frac{\mathrm{d} \mathbf{V}}{\mathrm{d} t}=-\operatorname{grad} p-\rho \operatorname{grad} U, \quad \frac{\mathrm{d}}{\mathrm{d} t}\left(\frac{p}{\rho^{\gamma}}\right)=0, \quad \Delta U=4 \pi G \rho,
$$

где $U$ - гравитационный потенциал, $G$ - гравитационная постоянная.

Предполагаем, что плоский слой толщины $h$ и с плотностью $\rho_{1}$ ускоряется под действием разности давлений на обеих его сторонах $\left(p_{0}-p_{2}\right)$ и для определенности $p_{0}>p_{2}$. Определяя характерное ускорение слоя $W$ как $W=\left(p_{0}-p_{2}\right) /\left(\rho_{1} h\right)$, введем параметр $\beta=2 \pi G \rho_{1} h / W$.

Считаем, что в некоторый момент времени в слое возникают возмущения вида

$$
u^{\prime}=\mathrm{A} \sin \left(\frac{2 \pi x}{\lambda}\right), v^{\prime}=\mathrm{A} \sin \left(\frac{2 \pi x}{\lambda}\right), \mathrm{A}=\mathrm{const},
$$

где $u^{\prime}, v^{\prime}-$ компоненты скорости по осям $x$ и $y ; \lambda$ - длина волны.

Установлено, что в области, где силы гравитационного притяжения между частицами среды больше или порядка разности давлений на сторонах слоя (то есть достаточно велик параметр $\beta$ ) структура конденсаций такова, что различия в масштабах неоднородностей в продольном и поперечном по отношению к невозмущенному движению направлениях значительно уменьшается по сравнению с неустойчивостью Рэлея-Тейлора. При этом учет самогравитации приводит к значительному увеличению максимального значения плотности в слое. Этот эффект иллюстрируется на рис. 3.
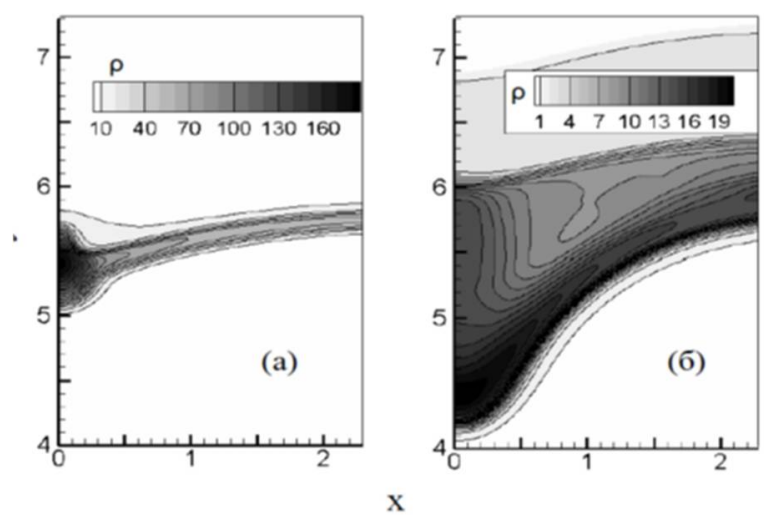

Рис. 3. Морфология уплотнений с учетом самогравитации для $\beta=3.1$ (а) и без учета самогравитации (б) в момент времени $t u_{0} /(L \sqrt{\gamma-1})=5$. Изохоры для движения с начальными условиями $\rho_{1} / \rho_{0}=25, \rho_{2} / \rho_{0}=1, p_{1} / p_{0}=1, p_{2} / p_{0}=0.04$. Параметры возмущений $\mathrm{A} \sqrt{\gamma-1} / L=-0.1, \lambda / L=5$

Здесь масштаб $L$ произволен. 
На основании расчетов показано, что возникновение неоднородной структуры области HII RCW 82 может быть обусловлено неустойчивостью оболочки, сформировавшейся при распространении ионизационно-ударного фронта, причем время развития неустойчивости меньше оцениваемого возраста области НII [19, 20].

\section{4. Проникновение облака в ускоренно движущийся газовый слой}

Результаты моделирования, приведенные в разделе 3, показывают, что проявления неустойчивостей ионизационно-ударного фронта, Рэлея-Тейлора или Рихтмайера-Мешкова морфологически отличаются мало - на внутренней границе слоя или оболочки образуются вытянутые в направлении к звезде уплотнения. В то же время внешняя граница слоя возмущена значительно меньше. Авторами настоящей работы впервые показано, что существенные изменения внешней границы слоя могут происходить при его движении в среде, содержащей локальные уплотнения [19,21]. Установлено, что при проникновении сферически симметричного облака в ускоренно движущийся слой образуется полость, которая со временем заполняется холодным газом, и при схлопывании которой возникает кумулятивная струя. Вещество струи истекает в направлении, противоположном направлению движения уплотнения. Однако в работах $[19,21]$ предполагалось, что до взаимодействия со слоем облако имело сферическую форму. Однако для рассмотренной в разделе 3 модели расширении областей Н II характерно присутствие ударной волны перед оболочкой. Следовательно, даже если изначально (до прохождения ионизационно-ударного фронта) облако имело сферическую форму, то она могла быть нарушена под действием ударной волны [22]. Поэтому целесообразно выяснить, какое влияние оказывает форма облака на кумулятивный эффект.

Как и в работах [19,21], рассмотрим упрощенную модель, согласно который сжатый и ускоренный ударной волной слой совершенного газа взаимодействует с изолированным уплотнением. При этом движение с читается адиабатическим.

Пусть имеется движущийся с ускорением $W$ плоский слой газа. Перейдем в систему отсчета, относительно которой слой покоится (т.е. находится в гравитационном равновесии) и занимает область «0» (рис. 4). Введем цилиндрическую систему координат z, r. Координату внутренней границы слоя примем равной $z=0$. Координату внешней границы слоя обозначим через $z_{c}$. Будем считать плоскость $z=z_{c}$ контактным разрывом, а располагающийся над ней при $z>z_{c}$ менее плотный газ также находящимся в равновесии (область «1»). Индексами «0» и «1» будем отмечать значения параметров газа на соответствующих сторонах контактного разрыва. Соответственно $\rho_{0}$ и $\rho_{1}-$ значения плотностей по обеим сторонам контактного разрыва; $L$ и $u_{\infty}$ - характерный пространственный масштаб движения и характерная скорость.

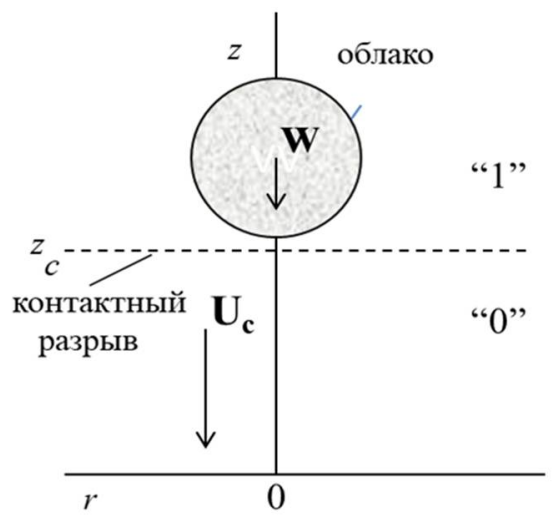

Рис. 4. Схема взаимодействия облака с плоским слоем 
Проникновение сгустка в слой «0» моделировалось численно путем задания в начальный момент времени в области $z \geq z_{c}$ осесимметричного распределения избыточных (по отношению к состоянию равновесия) плотности и скорости. В уравнении движения учитывалась массовая сила, обусловленная переходом в новую систему отсчета, связанную со слоем.

При проведении расчетов пространственным и временным масштабами служили $L$ и $L / u_{\infty}$. При этом $u_{\infty}$ выбиралось порядка начальной скорости облака $U_{c}$, а $L$ - порядка масштаба изменения плотности в области «0». Вводились безразмерные переменные

$$
\rho^{*}=\frac{\rho}{\rho_{1}}, \quad \mathbf{V}^{*}=\frac{\mathbf{V}}{u_{\infty}}, \quad E^{*}=\frac{E}{u_{\infty}^{2}}, \quad \varphi=\frac{(\gamma-1) W L}{\gamma a_{1}^{2}}, \quad a_{1}^{2}=\frac{p_{1}}{\rho_{1}}
$$

Далее на графиках индекс (*) у безразмерных переменных опускается. Использовалась монотонная разностная схема второго порядка аппроксимации.

Примем, что облако имеет эллипсоидальную форму.

Обозначим через $d_{z}$ и $d_{r}$ соответственно полуоси эллипсов по осям $z$ и $r$ соответственно. Координаты центров эллипсов $z=z_{d}, r=0$. Координата контактного разрыва $z=z_{c}$, «верхней» границы расчетной области $z=z_{b}$, левой $-r=r_{b}$. Полагаем, что плотность и скорость в облаке в начальный момент распределены по закону

$$
\rho-1=A_{d} \exp \left[-\frac{\left(z-z_{d}\right)^{2}}{d_{z}^{2}}-\frac{r^{2}}{d_{r}^{2}}\right], \quad v_{z}=B_{d} \exp \left[-\frac{\left(z-z_{d}\right)^{2}}{d_{z}^{2}}-\frac{r^{2}}{d_{r}^{2}}\right],
$$

где $A_{d}, B_{d}, z_{d}$ - постоянные.

Проведем сопоставление движений облаков одинаковой массы и обладающих также одинаковым полным импульсом.

Тогда для полной массы облака $M_{c}$ и его импульса $\Pi_{c}$ имеем выражения

$$
\begin{gathered}
M_{c}=2 \pi A_{d} \int_{z_{c}}^{z_{b}} \exp \left[-\frac{\left(z-z_{d}\right)^{2}}{d_{z}^{2}}\right] \mathrm{d} z \int_{0}^{r_{b}} \exp \left(-\frac{r^{2}}{d_{r}^{2}}\right) r \mathrm{~d} r, \\
\Pi_{c}=2 \pi A_{d} B_{d} \int_{z_{c}}^{z_{b}} \exp \left[-\frac{2\left(z-z_{d}\right)^{2}}{d_{z}^{2}}\right] \mathrm{d} z \int_{0}^{r_{b}} \exp \left(-\frac{2 r^{2}}{d_{r}^{2}}\right) r \mathrm{~d} r
\end{gathered}
$$

Введем обозначения

$$
\begin{gathered}
J_{r}=\int_{0}^{r_{b}} \exp \left(-\frac{r^{2}}{d_{r}^{2}}\right) r \mathrm{~d} r, J_{z}=\int_{z_{c}}^{z_{b}} \exp \left[-\frac{\left(z-z_{d}\right)^{2}}{d_{z}^{2}}\right] \mathrm{d} z, \\
J_{r}^{\Sigma}=\int_{0}^{r_{b}} \exp \left(-\frac{2 r^{2}}{d_{r}^{2}}\right) r \mathrm{~d} r, J_{z}^{\Sigma}=\int_{z_{c}}^{z_{b}} \exp \left[-\frac{2\left(z-z_{d}\right)^{2}}{d_{z}^{2}}\right] \mathrm{d} z
\end{gathered}
$$

Интегралы $J_{r}, J_{r}^{\Sigma}$ вычисляются точно, а $J_{z}, J_{z}^{\Sigma}$ сводятся к интегралу вероятности.

При фиксированных $d_{z}, d_{r}$ требованию постоянства $M_{c}, \Pi_{c}$ можно удовлетворить, варьируя коэффициенты $A_{d}, B_{d}$.

В качестве примера сопоставим результаты расчетов для сферического облака $\mathrm{d} z=\mathrm{d} r=0.3, z_{b}=7, z_{r}=3$ (вариант а) [21] - и для облаков, удлиненных в направлении $z$ (вариант б) и в направлении $r$ (вариант в).

Пусть для сферического облака $A_{d}=20, B_{d}=-0.5, \varphi=0.05, z_{c}=3, z_{d}=3.5$. Показатель адиабаты $\gamma=5 / 3$, плотность на контактном разрыве в области «0» $\rho_{c}=10$. Для варианта (б) 
примем $d_{z}=0.4, d_{r}=0.2$ (остальные параметры те же, что и для варианта а). Тогда, воспользовавшись приведенными выше выражениями для $J_{r}, J_{r}^{\Sigma}, J_{z}, J_{z}^{\Sigma}$, находим $A_{d}=34.76$; $B_{d}=-0.488$.

Полагая для варианта (в) $d_{z}=0.2, d_{r}=0.4$, будем иметь $A_{d}=16.71 ; B_{d}=-0.506$.

Изохоры, отвечающие движениям (a)-(в), изображены на рис. 5. Графики показывают, что при равенстве массы $M_{c}$ и импульса $\Pi_{c}$ хорошо выраженная кумулятивная струя возникает в том облаке, чей поперечный по отношению к направлению $U_{c}$ размер больше.
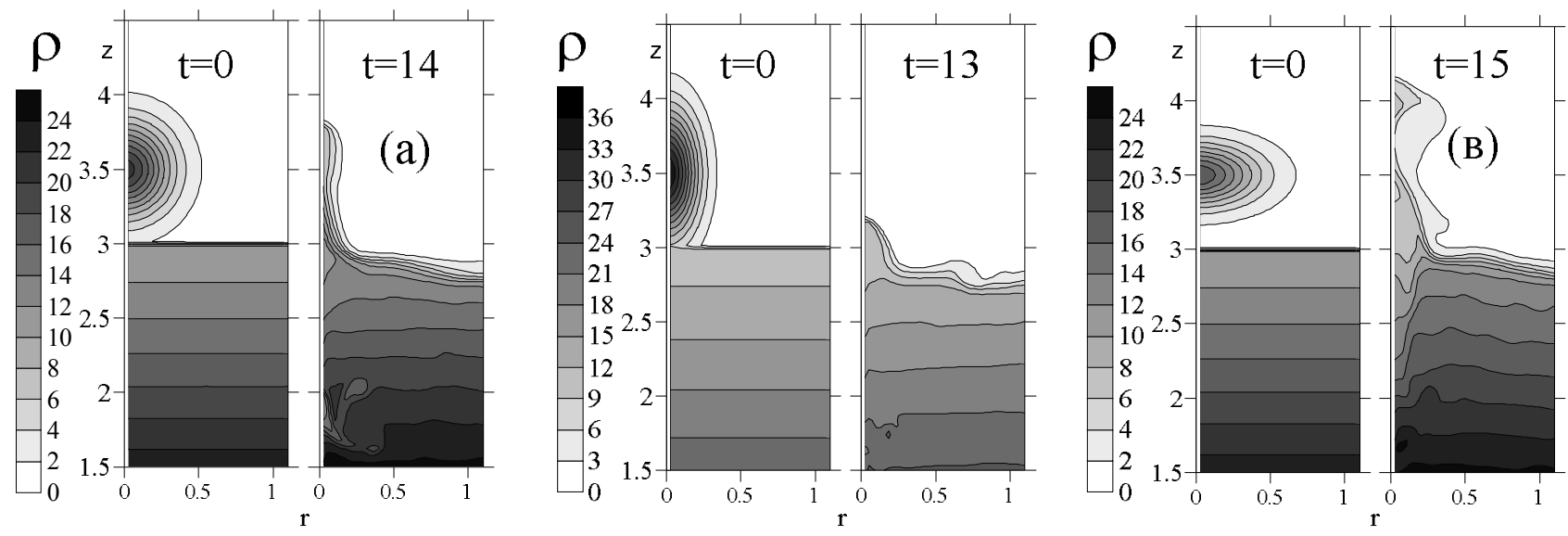

Рис. 5. Влияние формы облака на формирование струи

В целом полученные результаты моделирования позволяют по наблюдаемым параметрам оболочки и морфологии ее границы оценить параметры неоднородностей, существовавших в межзвездной среде до их взаимодействия с оболочкой.

\section{5. Заключение}

Недавние исследования с помощью космических аппаратов подтвердили теоретические представления о том, что наблюдаемые неоднородности плотности на периферии окружающего горячие звезды газа обусловлены гидродинамическими неустойчивостями. Компьютерное моделирование показало, что при развитии неустойчивостей могут создаваться условия для выполнения критерия Джинса. В результате возникающего гравитационного сжатия возможна фрагментация уплотнения и образование молодых звездных объектов. Исследование неустойчивостей ионизационно-ударного фронта, Рэлея-Тейлора и Рихтмайера-Мешкова позволило определить темп накопления массы в неоднородностях и оценить возраст молодых объектов. Впервые рассмотрена модель взаимодействия неоднородностей с ускоренно движущимся слоем, которая позволяет по наблюдаемым параметрам оболочки и морфологии ее границы приближенно установить параметры неоднородностей.

Работа выполнена при поддержке гранта РФФИ №18-01-00184.

\section{Литература}

1. Elmegreen B.G., Lada C.J. Sequential formation of subgroups in OB associations // Astrophys. J. 1977. Vol. 214. Pp. 725-741.

2. Churchwell E. et al. The bubbling Galactic disk. II. The inner $20^{\circ} / /$ Astrophys. J. 2007. Vol. 670. Pp. 428-441.

3. Deharveng L. et al. A gallery of bubbles. The nature of the bubbles observed by Spitzer and what ATLASGAL tells us about the surrounding neutral material // Astronomy \& Astrophysics. 2010. Vol. 523. Pp. 1-135. 
4. Anderson L.D. et al. The dust properties of bubble HII regions as seen by Herschel // Astronomy \& Astrophysics. 2012. Vol. 542. Pp. 1-27.

5. Aghanim N. et al. Planck intermediate results XXXIV. The magnetic field structure in the Rosette Nebula // Astronomy \& Astrophysics. 2016. Vol. 586. Pp. 1-16.

6. Capriotti, E.R. The structure and evolution of planetary nebulae // Astrophys. J. 1973. Vol. 179. Pp. 495-516.

7. White G. J. et al. The Eagle Nebula's fingers - pointers to the earliest stages of star formation? // Astronomy \& Astrophysics. 1999. Vol. 342. Pp. 233-256.

8. Schneider N. et al. Globules and pillars in Cygnus XI Herschel far-infrared imaging of the Cygnus OB2 environment // Astronomy \& Astrophysics. 2016. Vol. 591. A40. Pp. 1-21.

9. Mathews W.G., O'dell C.R. Evolution of Diffuse Nebulae // Annual Review of Astronomy and Astrophysics. 1969. Vol. 7. Pp. 67-98.

10. Баранов В.Б., Краснобаев К.В. Гидродинамическая теория космической плазмы М.: Наука, 1977. $335 \mathrm{c}$.

11. Спитцер Л. мл. Физика межзвездной среды М.: Мир, 1981. 351с.

12. Котова Г.Ю., Краснобаев К.В., Тагирова Р.Р. Двумерные неустановившиеся движения фотоиспаряемых газовых оболочек // Проблемы современной механики: к 85-летию академика Г.Г. Черного. 2008. М.: Изд-во МГУ, Омега-Л. С. 190-206.

13. Fedchenko A.S., Krasnobaev K.V. Analysis of the approximate solutions of HII region expansion problem // Journal of Physics: Conference Series. 2018. Vol. 1129.

14. Котова Г.Ю., Краснобаев К.В. Ускорение сферической нейтральной оболочки, формируемой ионизационно-ударным фронтом в неоднородной межзвездной среде // Письма в Астрономический журнал. 2009. Т. 35. № 3. С. 189-198.

15. Котова Г.Ю., Краснобаев К.В. Численное моделирование неустойчивых двумерных движений околозвездной оболочки // Письма в Астрономический журнал. 2010. Т. 36. № 7. С. 506-516.

16. З Зоненко С.И., Черный Г.Г. Новый вид кумуляции энергии и импульса метаемых взрывом пластин и оболочек // Доклады РАН. 2003. Т. 390. №1. С. 46-50.

17. Falle S.A.E.G. A numerical calculation of the effect of stellar winds on the interstellar medium // Astronomy and Astrophysics. 1975. Vol. 43. №. 3. Pp. 323-336.

18. Краснобаев К.В., Тагирова Р.Р. О проявлении неустойчивости Рихтмайера-Мешкова в неоднородной межзвездной среде с высвечиванием // Письма в Астрономический журнал. 2009. Т. 35. № 5. С. 364-371.

19. Краснобаев К.В., Котова Г.Ю., Тагирова Р.Р. Двумерные возмущения ускоренного движения неоднородных газовых слоев и оболочек в межзвездной среде // Письма в Астрономический журнал. 2015. Т. 41. № 3-4. С. 123-132.

20. Krasnobaev K.V., Tagirova R.R., Kotova G.Y. Model of the expansion of HII region RCW 82 // Astrophys. J. 2014. Vol. 786. № 2. Pp. 90-95.

21. Kotova G.Y., Krasnobaev K.V. Interaction of an accelerating layer with a cloud: formation of tails and cumulative jets // Monthly Notices of the Royal Astronomical Society. In Press.

22. Pittard J.M. et al. The turbulent destruction of clouds - I. A k-epsilon treatment of turbulence in 2D models of adiabatic shock-cloud interactions // Monthly Notices of the Royal Astronomical Society. Vol. 394. № 3. Pp. 1351-1378. 